\title{
SEgmentation of Cysts in Kidney AND 3-D Volume Calculation From CT IMAgES
}

\author{
Nanzhou Piao, Jong-Gun Kim, and Rae-Hong Park \\ Department of Electronic Engineering, School of Engineering, Sogang University \\ 35 Baekbeom-ro, Mapo-gu, Seoul, 121-742, Korea
}

\begin{abstract}
This paper proposes a segmentation method and a three-dimensional (3-D) volume calculation method of cysts in kidney from a number of computer tomography $(C T)$ slice images. The input CT slice images contain both sides of kidneys. There are two segmentation steps used in the proposed method: kidney segmentation and cyst segmentation. For kidney segmentation, kidney regions are segmented from CT slice images by using a graph-cut method that is applied to the middle slice of input CT slice images. Then, the same method is used for the remaining CT slice images. In cyst segmentation, cyst regions are segmented from the kidney regions by using fuzzy $C$-means clustering and level-set methods that can reduce noise of non-cyst regions. For 3-D volume calculation, cyst volume calculation and 3-D volume visualization are used. In cyst volume calculation, the area of cyst in each CT slice image equals to the number of pixels in the cyst regions multiplied by spatial density of CT slice images, and then the volume of cysts is calculated by multiplying the cyst area and thickness (interval) of CT slice images. In 3-D volume visualization, a 3-D visualization technique is used to show the distribution of cysts in kidneys by using the result of cyst volume calculation. The total 3-D volume is the sum of the calculated cyst volume in each CT slice image. Experimental results show a good performance of 3-D volume calculation. The proposed cyst segmentation and 3-D volume calculation methods can provide practical supports to surgery options and medical practice to medical students.
\end{abstract}

\section{KEYWORDS}

Computer Tomography, Slice Images, Image Segmentation, 3-D Visualization, Kidney Segmentation, Cyst, Cyst Segmentation, Graph-cut, Level-set Method, Fuzzy C-means Clustering, Volume Calculation.

\section{INTRODUCTION}

Medical images, acquired from different imaging modalities such as computer tomography (CT), $\mathrm{X}$-ray imaging computed tomography (X-ray CT), positron emission tomography (PET or $\mathrm{PET} / \mathrm{CT}$ ), and magnetic resonance imaging (MRI), become important components in routine clinical applications. Image processing and computer vision techniques [1-8] are applied to various medical images to improve the accuracy of medical diagnosis and thus to provide useful information to doctors for surgical operations or routine treatments.

Medical image processing techniques have been developed to segment or detect lung cancer, liver tumor, and cyst [1]. Existing medical image processing techniques use image segmentation methods to segment organ regions, tumor regions or cyst regions from CT slice images [1-3]. In addition, image segmentation methods can be used to locate the boundaries of organ regions or cyst regions [6-8]. More precisely, they classify labels of pixels in CT slice images that have the same or similar intensity values or share certain visual characteristics. Meanwhile, since medical

DOI : $10.5121 /$ ijcga.2015.5101 
image processing techniques can provide valuable information of patients, a lot of useful data can be used in disease research and other medical applications.

Medical image technology has been revolutionized by combining with image based threedimensional (3-D) visualization techniques. These 3-D visualization technologies can observe the hidden details of organs, such as the 3-D volume calculation and the distribution of cysts [9], [10]. An existing volume calculation method [1] is a fairly simple but efficient way to measure the volume of organ by counting the numbers of pixels in the organ regions. However, the volume of organ in each CT slice image cannot provide too much important information, such as the distribution of organ and the preferred direction of surgical operation. By using 3-D visualization to reconstruct volume of organs or cysts, the distribution and volume can be easily observed. Combined with 3-D visualization, the information of calculated volumes that can be provided is beyond the numbers. The 3-D volume is reconstructed by using the information of medical imaging data set. These data sets are acquired by various imaging modalities such as MRI scanners or CT scanners. 3-D visualization techniques create a virtual reality of a real object with 3-D digital reconstruction and interaction [9], [10]. 3-D visualization of medical images uses a stack of two-dimensional (2-D) image slices to reconstruct a 3-D image model, which can bring a lot of important medical information to surgery and cancer treatment in developing medical technology. Image analysis based 3-D visualization using various image processing and computer vision methods can improve the accuracy of image data set [9], [10]. 3-D volume visualization is a current trend of medical imaging techniques for routine diagnosis and treatment procedures [10]. In medical applications, the distribution and calculated volume of cysts can give a lot of convenience to the surgery and reduce the pain of patients. In education, it allows medical school students to examine human anatomy for simulation and training. This approach will enable to create medical datasets that bring a greater understanding in anatomy knowledge and provide important information in anatomical research and training.

Cysts have a saclike structure which is full of hazardous substances, such as fluid, gas, and particles. Materials in cysts can be changed to semisolid objects, which become tumor or cancer. This is how carcinomatous change occurs. The structures of cysts like footballs have an inner wall that is full of hazardous substances and an outer wall that is contacted to other organs. Cysts can exist anywhere in the body. Most of them can only be detectable by a microscope or in CT slice images. They can grow very large, thus sometimes displace normal organs and tissues. However, experts do not use an image-based segmentation method and instead calculate the total volume of cysts manually. Cysts in kidney usually show no signs or symptoms, and there are many cysts in one kidney. Also, distribution of cysts in kidney is random. With these properties, cyst volume is difficult to be calculated. Cyst volume can provide the condition of patients because expansion of cyst volume will bring a lot of pain or damage to other organs. Volume of cysts not only provides useful information to experts for surgical operations, but also gives valuable information for disease research. So cyst volume calculation is important and this process needs a high cost with unstable segmentation result. To solve this drawback, a graph-cut method [1] was proposed for kidney segmentation and semi-automatic volume measurement methods for kidney region. Segmentation methods for semi-automatic liver tumor segmentation were proposed using fuzzy C-means clustering [2] and level-set methods [3]. However, relatively few researches have been focused on cyst segmentation and 3-D volume calculation of cysts.

In this paper, a segmentation method and a 3-D volume calculation of cyst regions in kidney are proposed. The graph-cut method [1] is used for kidney segmentation to segment the kidney region whereas both fuzzy C-mean clustering [2] and level-set method [3] are used to segment cyst regions. After cyst segmentation, the total volume of cysts in kidneys is calculated. In order to facilitate observation of segmented cysts, 3-D visualization [9], [10] is done. 
The rest of the paper is organized as follows. In Section 2, a segmentation method and 3-D volume calculation method are proposed. Also 3-D visualization of cysts is addressed. In Section 3 , experimental results and discussions are presented. Conclusion and future work are given in Section 4.

\section{Segmentation AND 3-D Volume Calculation}

In order to have accurate regions of cysts, segmentation methods are used. The region of cysts can be detected by using segmentation methods [1-3]. After extracting the regions of cysts, volume calculation is performed. With the volumes of cysts, a 3-D volume model [9], [10] of cysts is reconstructed. Segmentation methods such as graph-cut method [1], fuzzy C-means clustering method [2], and level-set method [3] are presented in each step of the proposed method in Section 2.1. The process of cyst volume calculation with given information of input CT slice images is described in Section 2.2. 3-D volume visualization is presented in Section 2.3.

\subsection{Segmentation Methods}

The proposed segmentation method consists of two steps to reduce the interest regions of cysts. The first step is kidney segmentation, in which a graph-cut method [1] is used to segment the kidney regions from input CT slice images. The second step is cyst segmentation, which segments cyst regions from kidney regions by using fuzzy C-means clustering [2] and level-set method [3]. In a CT slice image, there are kidneys and some other organs. Kidney segmentation is performed as pre-processing to reduce the region of interest. In order to segment cyst regions from kidney regions, cyst segmentation is performed.

Figure 1 shows a set of input CT slice images [1], in which gray regions represent the human body and the regions within white contours represent the kidney regions on each side of the body. In the middle slice, the red and blue ellipses denote cysts in the right and left kidneys, respectively. The green circles represent non-cyst regions between cysts. Let there be $N$ slices of CT slice images, with the same thickness and the kidney be separated as left and right kidneys in a CT slice image. In practice, cysts may not exist across several CT slice images, because a lot of cysts are randomly distributed in the kidney. Therefore, some of CT slice images may not have cysts. The segmented kidney regions and cyst regions are stored for volume calculation and 3-D volume visualization of cysts.

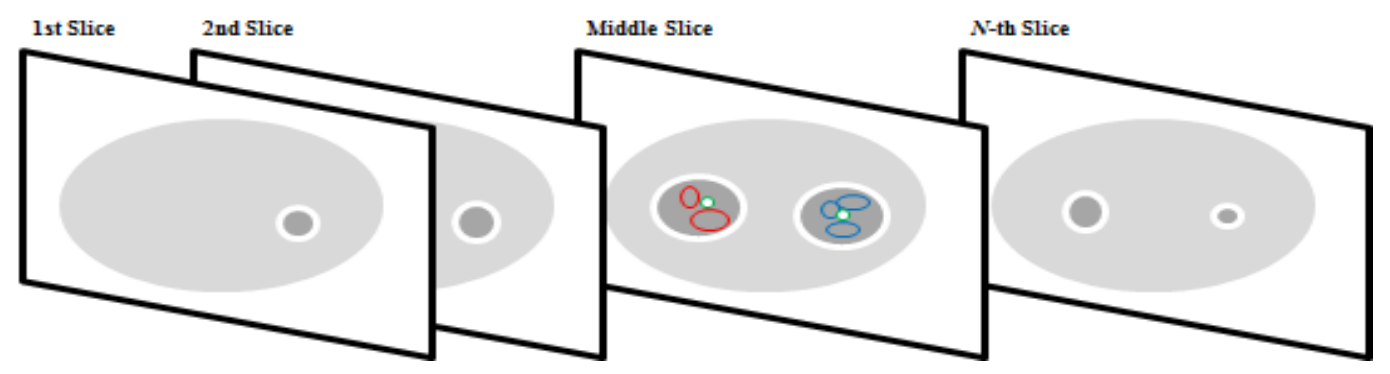

Figure 1. Input CT slice images. Each CT slice image is assumed to be taken with the same thickness. The gray regions represent human body. The white, red, blue, and green contours denote the boundaries of the kidney, cysts in the right kidney, cysts in the left kidney, and non-cyst regions, respectively. 
International Journal of Computer Graphics \& Animation (IJCGA) Vol.5, No.1, January 2015

\subsubsection{Kidney Segmentation}

In kidney segmentation, the first or last CT slice image may not contain the kidney region to be segmented. So the middle CT slice image is chosen and a contour is drawn manually around the kidney to initialize kidney segmentation. A graph-cut method [1] is used to segment the kidney region in the middle CT slice image with the initialized kidney contour. After that, the remaining CT slice images are processed by the same method using the kidney region extracted from the middle CT slice image. If the segmentation result of the kidney region is not good enough (for example, other organs or tissues can be included in the kidney region after kidney segmentation), the kidney contour should be modified by adding the hard constraint (HC) to increase the segmentation accuracy. This approach is called graph cuts based active contours (GCBAC) [5]. The GCBAC algorithm dilates the object contour into neighboring contour region. Vertices of inner and outer contours of neighborhood region are identified and set as source and sink, respectively. A new optimal contour is detected by s-t computing minimum cut [5] between extracted source and sink. Another goal of the GCBAC algorithm is using HC to guide the resulting contour to follow the true object contour, in order to obtain an optimal contour and thus to increase the accuracy of kidney segmentation.

Figure 2 shows $\mathrm{HC}$ applied on the current contour that is obtained using the GCBAC algorithm [5]. The black dotted contours represent the contour of kidneys detected by the GCBAC algorithm. The contour of kidneys is dilated to generate the white region between blue and green contours called contour neighborhood region, where the green contour and blue contour represent the outer contour and inner contour of the neighborhood region, respectively. The red dot denotes the location where $\mathrm{HC}$ is applied. The location of applied $\mathrm{HC}$ can guide the graph-cut [1] process to follow the real contour of the kidney region to increase the accuracy of kidney segmentation. Figure 2(a) shows the contour representation after graph-cut segmentation. The processes of applying $\mathrm{HC}$ to the result of a graph-cut method update the source and sink nodes by stretching the inner and outer contours to obtain a new contour that belongs to a narrow gap between the stretched inner or outer contours. There are three ways to apply HC. In Figure 2(b), HC is used to correct the small error of the contour, in which the $\mathrm{HC}$ is applied between inner and outer contours. Then, the resulting contour obtained by a graph-cut method follows the correct contour to accurately segment the kidney region. Figures 2(c) and 2(d) show the HCs applied to the inner contour and the outer contour, respectively. The black dotted lines in Figures 2(b), 2(c), and 2(d) are the kidney contours extracted by the GCBAC algorithm [5]. Kidney contours estimated by adding $\mathrm{HC}$ are used to reconstruct kidney contours. Experimental results of kidney segmentation are shown in Section 3.

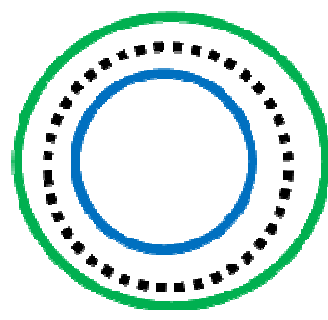

(a)

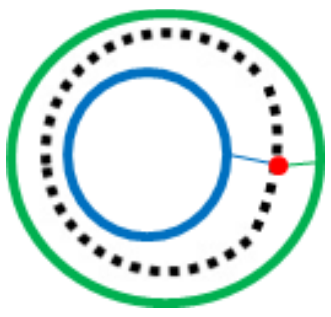

(b)

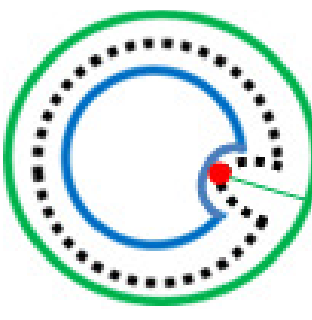

(c)

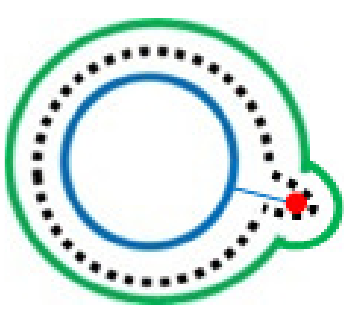

(d)

Figure 2. HC applied on the contour. Black dotted contours represent the kidney contours. (a) contour representation after graph-cut segmentation, (b) HC applied between inner and outer contours, (c) HC 
applied to the inner contour, (d) HC applied to the outer contour. Black dotted contours in (b), (c), and (d) are the kidney contours detected after adding HC.

\subsubsection{Cyst Segmentation}

In cyst segmentation, cyst regions are segmented from the kidney regions by using fuzzy Cmeans clustering [2] and level-set method [3]. Fuzzy C-means clustering is a popular algorithm and widely applied to medical image processing [2], [3] to classify the region of interest. In order to segment objects into different clusters, fuzzy C-mean clustering uses the membership degree of an object [2]. Since the intensities of cysts and kidneys are usually different from each other, intensities are considered as membership values. Cyst regions are found by comparing mean intensity values of three different clustered regions in kidneys: normal kidney region, cyst region, and back-ground region. The mean intensity is computed as

$$
\bar{p}^{k}=\frac{1}{N} \sum_{i=1}^{N} p_{i}^{k}
$$

where $p_{i}^{k}$ denotes the intensity value of the $i$-th pixel in the $k$-th cluster, $N$ represents the number of input CT slice images, and $\bar{p}^{k}$ signifies the mean intensity value of pixels in the $k$-th cluster.

After fuzzy C-means clustering, non-cyst regions contain some noises in resulting images of cyst segmentation. Since the distributions of cysts are rather random, some non-cyst regions in the cyst regions may not be segmented correctly. Non-cyst regions contain the normal kidney region and some other tissues in kidney. Meanwhile, non-cyst regions contain some noise in the inner contour of segmented cyst regions. To reduce this drawback, the level-set method [3] is used to suppress noises of non-cyst regions and to improve the performance of cyst segmentation. The level-set method can integrate the information of an image and properties of a model for optimal cyst segmentation. Typical edge-based level-set method uses a constant balloon force to control the dynamic interface of a model. The level-set method [3] uses a signed balloon force to segment the real contour of cysts. This constant force is modulated by the edge indicator. Resulting images of cyst segmentation are shown in Section 3.

\subsection{Cyst Volume Calculation}

In cyst volume calculation, the cyst area in a single CT slice image is calculated by multiplying the number of pixels within cyst area and the given density unit of CT slice images. The number of pixels in cyst areas can be calculated by subtracting the number of pixels within non-cyst regions from that within cyst regions. Non-cyst regions and cyst regions are specified by the cyst segmentation results, as described in Section 2.1. It is assumed that pixels within non-cyst regions correspond to the normal kidney or other organ tissues in cyst regions. Therefore, the pixels surrounded by the cyst regions include pixels within actual cyst regions and non-cyst regions.

In the existing organ volume calculation method [1], the organ volume is calculated by multiplying the segmented organ areas and the given thickness of CT slice images. The organ areas are calculated by multiplying the given density of CT slice images and the number of pixels in the regions of organs. After calculating the volume of an organ in a single CT slice image, the total volume of an organ is calculated by adding organ volumes of all CT slice images together. Therefore, the cyst volume of each CT slice image can be calculated by multiplying the cyst area and thickness. The total cyst volume in all CT slice images are calculated by the sum of all cyst volumes, i.e., 


$$
V_{D}=\left(\sum_{k=1}^{N} N_{D}^{k}\right) \times m \times t
$$

where $V_{D}$ represents the total volume of cysts with $D$ denoting left or right kidney $(D=\{L, R\})$. $N_{D}^{k}$ signifies the number of pixels in cyst regions in the $k$-th CT slice image, which can be calculated as $N_{D}^{k}=N_{\text {out }}^{k}-N_{\text {in }}^{k}$, where $N_{\text {in }}^{k}$ and $N_{\text {out }}^{k}$ signify the numbers of pixels within the inner contour and outer contour in the $k$-th CT slice image, respectively. In the case that some CT slice images do not contain cysts, the number of pixels within the cyst region equals to 0 , i.e., $N_{D}^{k}=0$. $k$ is the index of CT slice images, $N$ represents the total number of input CT slice images, $m$ is the density of CT slice images, with the unit of millimeter per pixel $(\mathrm{mm} / \mathrm{pixel})$, and $t$ denotes the thickness between two adjacent CT slice images.

\subsection{3-D Volume Visualization}

3-D volume visualization uses information of cysts in CT slice images to show the distributions and volume of cysts in 3-D spatial visualization. 3-D visualization techniques used for medical imaging procedures have been presented [8]. These techniques combine 2-D CT slice images to reconstruct 3-D representation of cysts. The volume of cysts can be observed as a byproduct of 3D reconstruction. 3-D visualization uses the results of cyst segmentation along with cyst volume calculation in each CT slice image. The CT slice images that do not have cysts are used to reconstruct the 3-D volume of cysts. The 3-D volume visualization of cysts are represented as the combined array with the sequence of all CT slice images, i.e.,

$$
V=\left\{V_{D, k}\right\}, \quad 1 \leq k \leq N
$$

where $V$ represents the reconstruction result of 3-D volume of cysts, $k$ denotes the sequence index of input CT slice images, and $V_{D, k}$ is the total cyst volume in the $k$-th CT slice image. The reconstruction process puts the cyst volumes of all CT slice images into a sequence to perform the 3 -D volume visualization.

\section{EXPERIMENTAL RESULTS AND DISCUSSIONS}

Experimental results of kidney segmentation, cyst segmentation, cyst volume calculation, and 3D volume visualization of cysts in patient's CT slice images are presented and discussed in this section. A flowchart of each process is shown in Figure 3. In kidney segmentation, the kidney regions are segmented from input $\mathrm{CT}$ slice images. The segmented kidney regions are used as the input of cyst segmentation to classify the cyst regions. After cyst volume calculation, the volumes of cysts in each CT slice image are listed in the volume calculation report. Based on the results of cyst segmentation and cyst volume calculation, 3-D volume visualization of cysts is performed. The system is processed by Image J program [4] which is built in the Java environment. The used information of patient and photographic equipment for volume calculation are in the header of digital imaging and communications in medicine image (DICOM).

\subsection{Kidney Segmentation}

Figure 4 shows the process of kidney segmentation, along with experimental results of the middle CT slice image. Figure 4(a) shows kidney contour initialization. The yellow contour is 

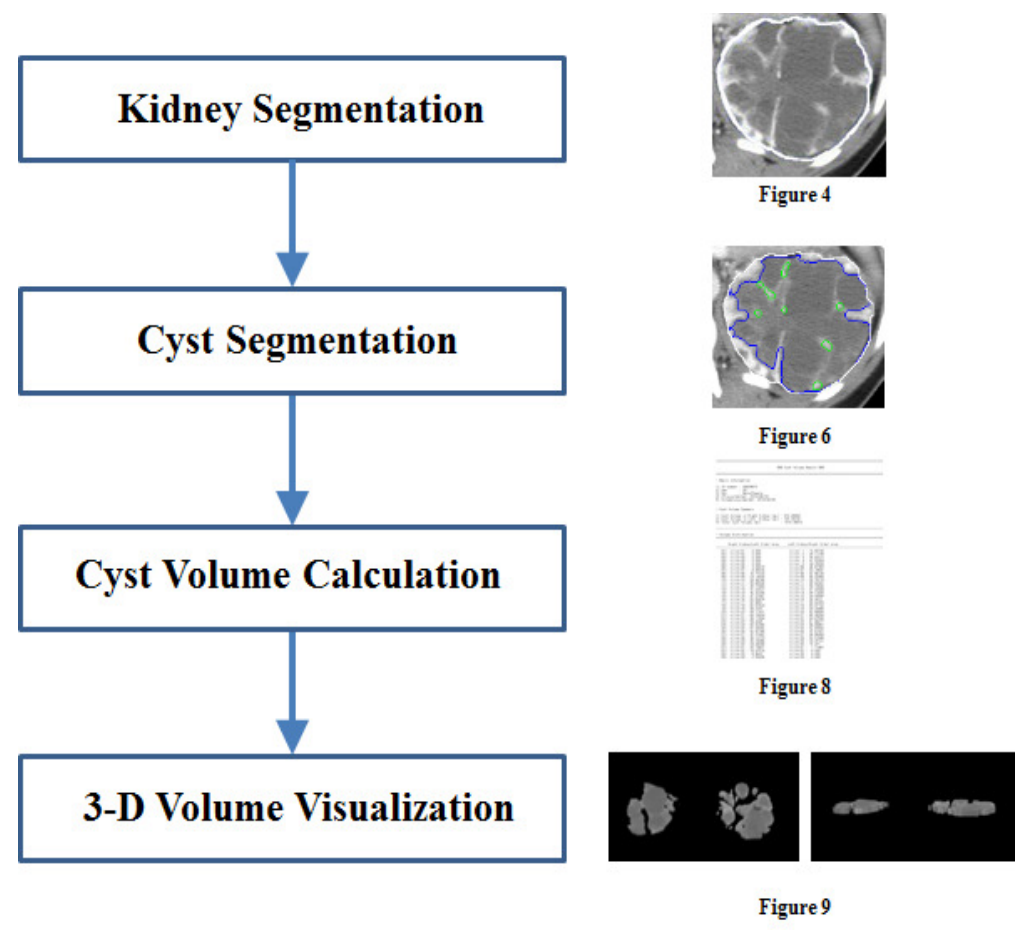

Figure 3. Flowchart of the proposed method and experimental results. Functions of the blocks in the left side are the processes of the proposed method, with the results of each process shown in the right side.

the initial contour that is drawn manually. Figure 4(b) shows the result of kidney segmentation (white contour) using the GCBAC algorithm [5] with the initial kidney contour (yellow contour). In Figure 4(c), the white region, gray region, and black region enclosed by black circles are the ribs, normal kidney tissue, and empty space between organs near the initial contour, respectively. $\mathrm{HC}$ is applied on the contour within these black circles. Figure 4(d) shows the result of applying $\mathrm{HC}$ on the contour to increase the accuracy of kidney segmentation. The white contour represents the result of kidney segmentation using the GCBAC algorithm [5] whereas the red contour denotes an estimated kidney contour after applying HC, which guides the contour to separate the non-kidney regions from the kidney regions. Figure 4(e) is the final result of kidney segmentation after adding HC. The kidney segmentation result shows a high accuracy of kidney regions by comparing Figures 4(c) and 4(e), in which the kidney contour is accurately extracted (see the regions enclosed by black circles on the boundary of kidney).

\subsection{Cyst Segmentation}

Figure 5 shows the process of the fuzzy C-means clustering for cyst region segmentation, in which three different regions (kidney, cyst, and background regions) are indicated in white (in (b), (c), and (d), respectively). In right column, histograms of corresponding regions are shown. Figure 5(a) shows the kidney region after performing kidney segmentation. In order to use fuzzy $\mathrm{C}$-means clustering [2], the kidney region is cropped to reduce the region of interest by modifying the intensity value of pixels, in which intensity values of the pixels of non-kidney regions are set to zero. Figure 5(b) shows the normal kidney, which has the largest mean intensity value. Figure 5 (c) illustrates the cyst region, where the regions having the middle mean intensity value are 
chosen as the regions of cysts. Figure 5(d) shows the background region that has the smallest mean intensity value.

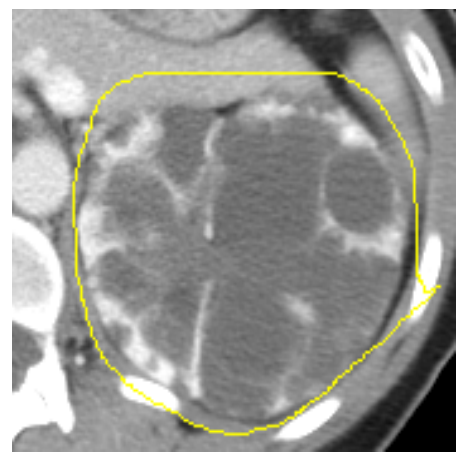

(a)

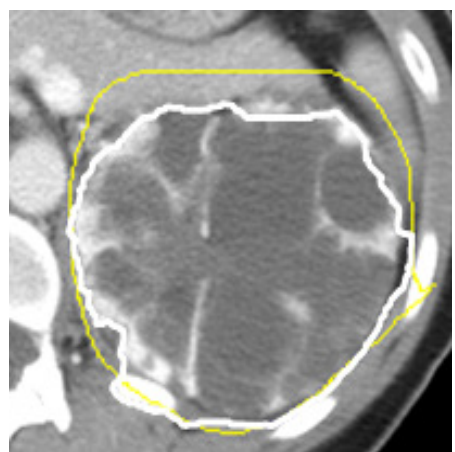

(b)

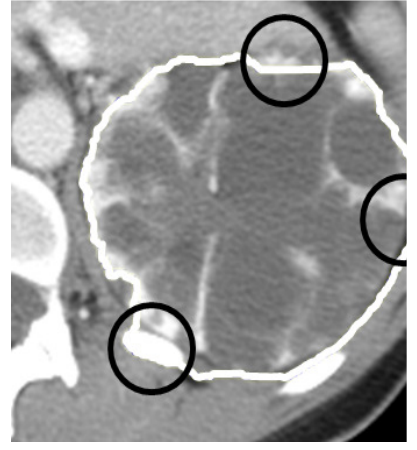

(c)

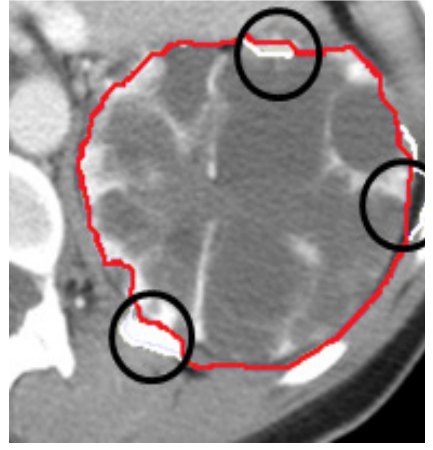

(d)

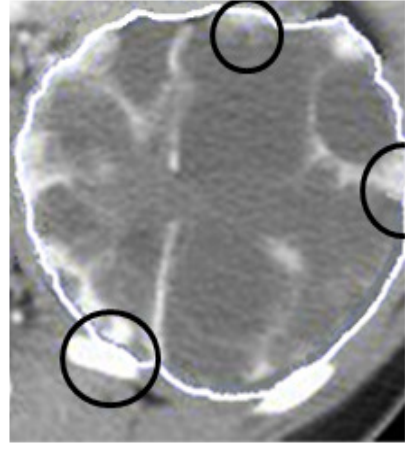

(e)

Figure 4. Process of kidney segmentation. (a) kidney contour initialization, (b) result of kidney segmentation using the graph-cut method, (c) HC applied to kidney contour, (d) result of HC applied to kidney segmentation, (e) final result of kidney segmentation with HC. The red contour in (d) denotes an estimated kidney contour and the white contour in (e) represents the final kidney contour.

Figure 6 shows the process of cyst segmentation using the level-set method. Figure 6(a) shows the result of kidney segmentation. The white contour is the kidney contour extracted by using the GCBAC algorithm [5]. The white regions enclosed by orange dotted ellipses are non-cyst regions that are to be segmented by cyst segmentation using fuzzy C-means clustering. Figure 6(b) shows the result of cyst segmentation using fuzzy C-means clustering. Blue and red contours are the cyst contours. A lot of non-cyst regions have been segmented by comparing the cyst contour and kidney contour. In Figure 6(c), the darker region within the cyst contour represents the cyst region. Meanwhile, there are still lots of non-cyst regions in the cyst region, as mentioned in Section 2.2, such as the regions in magenta dotted ellipses. Figure 6(d) shows the result of cyst segmentation using the level-set method. The regions in green contour represent non-cyst regions between cysts. The area of the cyst regions can be calculated by subtracting that of the regions within the green contour from that of the regions within the blue contour. 
Figure 7 shows the result of segmentation method applied to CT slice images of the same patient. Figures 7(a) and 7(b) show the 19-th and 20-th CT slice images that have been chosen as examples. The white contour represents the kidney contour of both kidneys. The cysts in both sides of kidneys have been segmented and discriminated by the red contour and blue
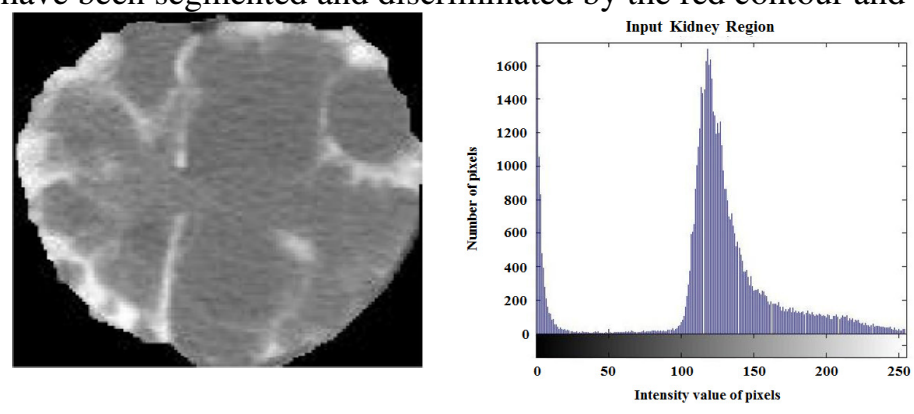

(a)
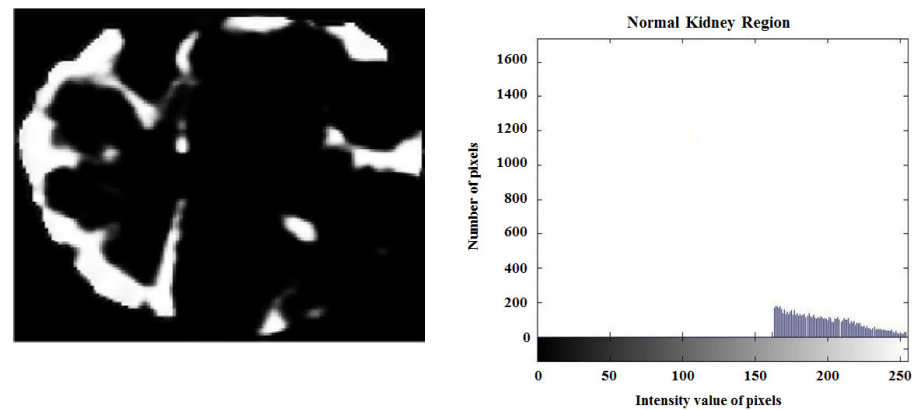

(b)
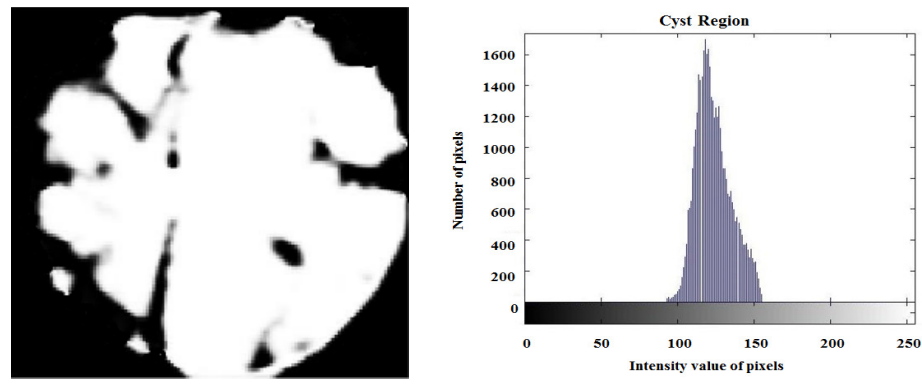

(c)
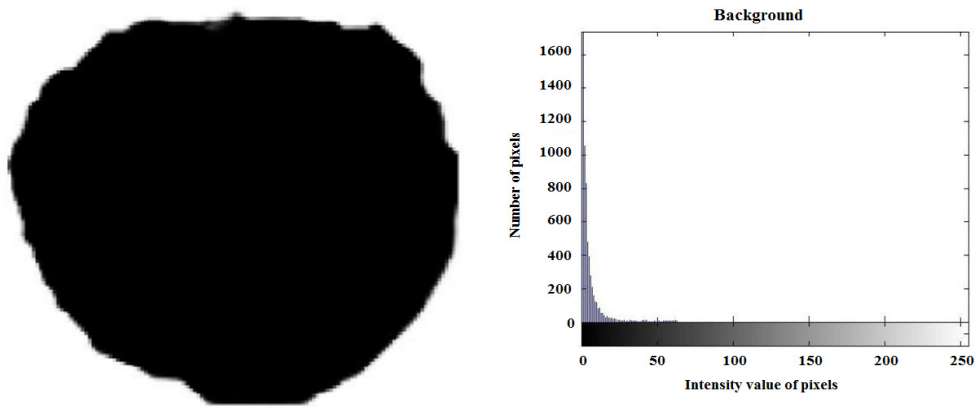

(d) 
Figure 5. Process of cyst segmentation using fuzzy C-means clustering. The right and left columns show the process of fuzzy C-means clustering and the histogram corresponding to each region, respectively. Three regions (normal kidney region, cyst region, and background region) are indicated in white (in (b), (c), and (d), respectively), (a) input kidney image, (b) normal kidney region, (c) cyst region, (d) background region.

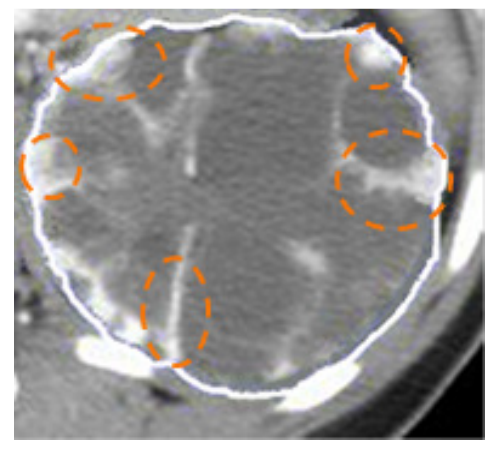

(a)

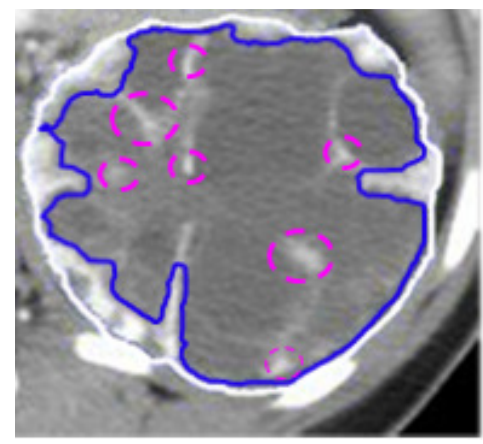

(c)

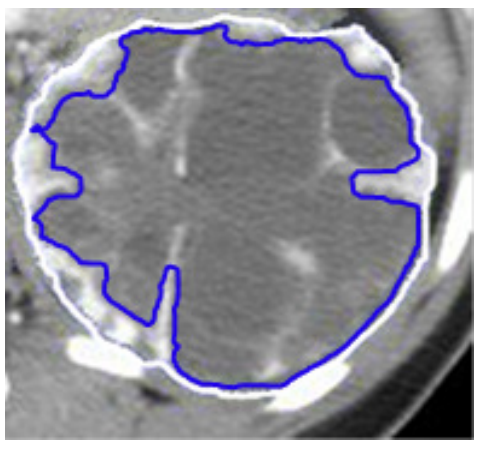

(b)

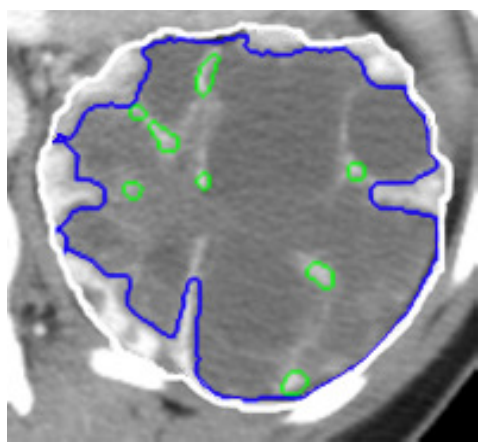

(d)

Figure 6. Process of cyst segmentation using the level-set method. (a) result of kidney segmentation, (b) result of cyst segmentation (fuzzy $\mathrm{C}$-means clustering), (c) non-cyst regions existing in cyst regions, (d) result of cyst segmentation (level-set method).

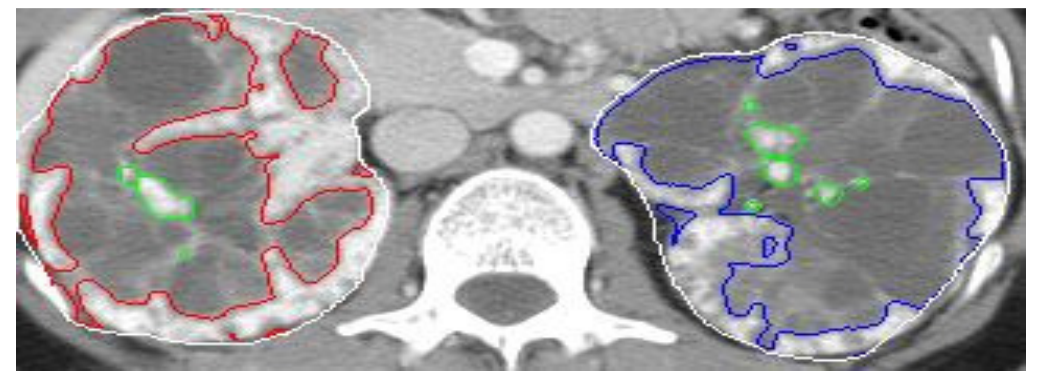

(a) 


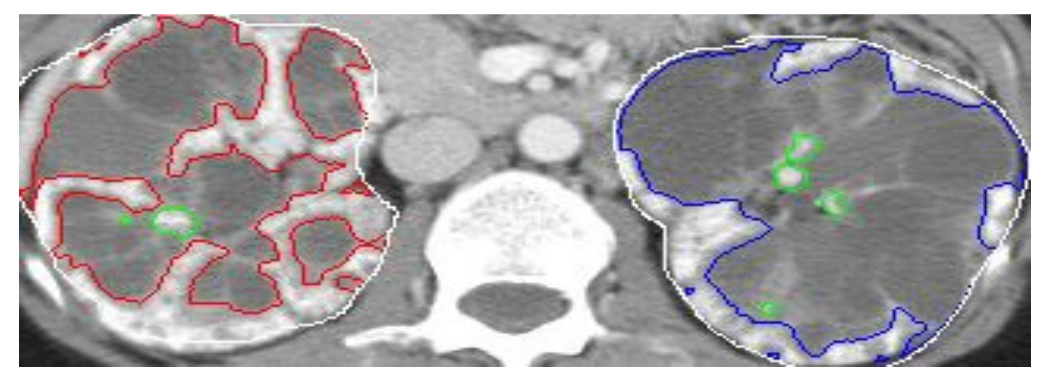

(b)

Figure 7. Result of segmentation superimposed on CT slice images. Two CT slice images are chosen as examples. (a) 19-th CT slice image, (b) 20-th CT slice image.

contour to distinguish the left kidney and right kidney in CT slice images, respectively. The region within the green contour is the non-cyst region.

\subsection{Cyst Volume Calculation}

After segmentation methods applied to CT slice images, volume calculation is processed. The first step is to calculate the cyst area in a CT slice image. In Figure 7, the pixels within green contour and blue (red) contour belong to non-cyst regions and cyst regions, respectively. The number of pixels in cyst area is calculated by subtracting the number of pixels in the green contour from the number of pixels in the blue (red) contour, as mentioned in Section 2.2. The volume calculation is processed only in the CT slice images that contain cysts, and the calculation can handle either continuous or discrete input CT slice images with cyst regions.

Figure 8 shows the report of cyst volume calculation using the segmentation result of all CT 


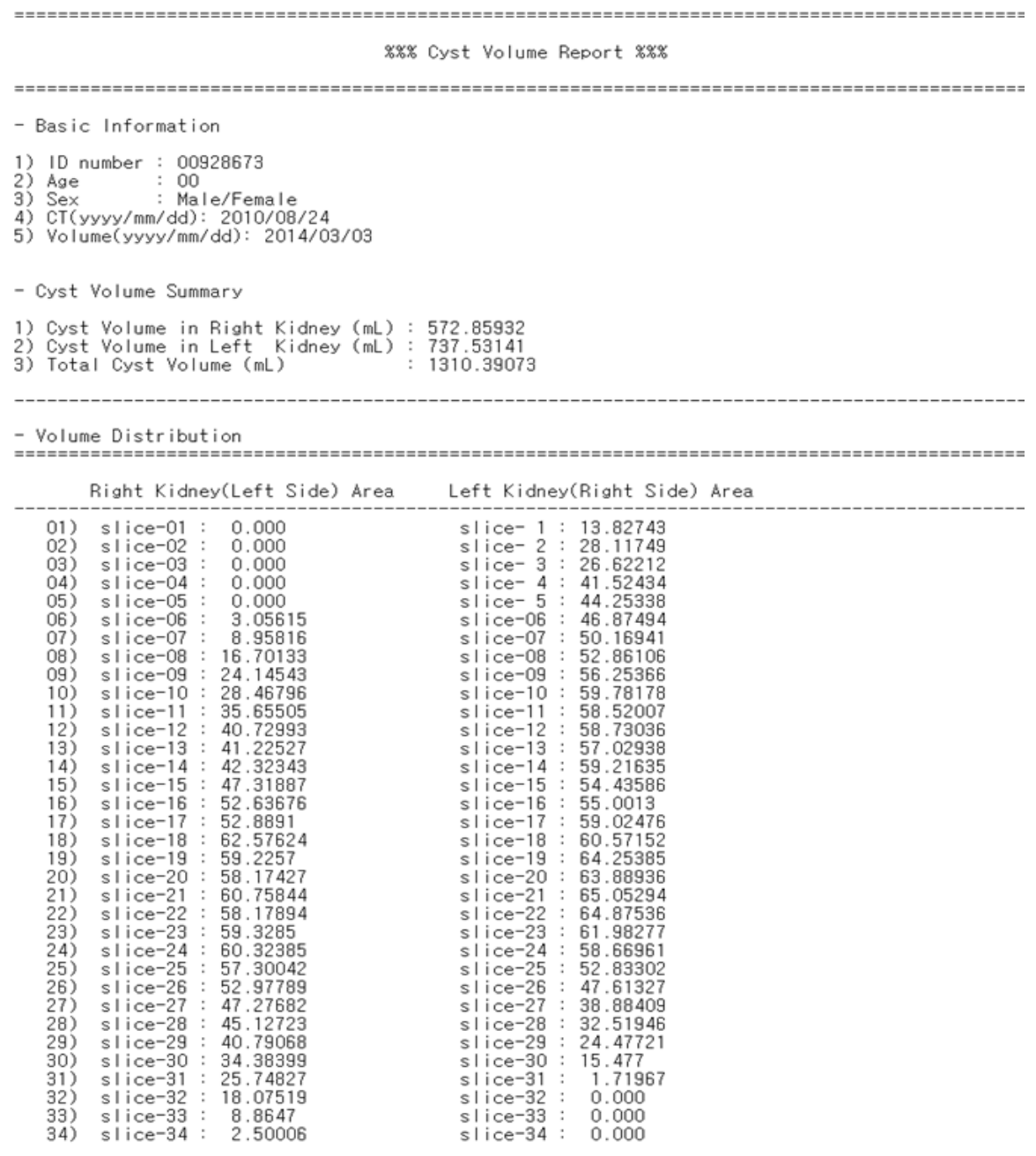

Figure 8. Cyst volume report. There are three parts listed in the report: basic information, cyst volume summary, and volume distribution. In the volume distribution, the number in parentheses represents the index of CT slice images and the following numbers are cyst volume values in CT slice images.

slice images. The thickness and the density of CT slice images are $5 \mathrm{~mm}$ and $0.6836 \mathrm{~mm} / \mathrm{pixel}$, respectively. There are three parts in the report: the basic information of input CT slice images, cyst volume summary, and the volume distribution of cyst. In the basic information, the ID number, age, and sex represent the identification of the patient. The CT (yyyy/mm/dd) signifies the data acquisition date of input CT slice images. Volume (yyyy/mm/dd) denotes the date of cyst volume calculation. In the cyst volume summary, the volume of cysts in the right kidney, volume of the left kidney, and the total volume of cysts are listed. The total cyst volume is the sum of cyst volumes in the right and left kidneys. At the bottom part of the report, the volumes of cysts in each side and both sides of kidneys are separately listed. The CT slice images are taken in the opposite direction. Thus, right kidney areas represent the cyst volumes in the left kidney in the CT slice images, whereas the left kidney areas are related to the cyst volumes in the right kidney. This process of the cyst segmentation and volume calculation can be repeatedly performed. Details of the cyst volume report can provide some useful information to experts for surgical 
operations. However, the distribution of cysts and some hidden information are hard to be observed. Therefore, 3-D visualization is useful.

\subsection{3-D Volume Visualization}

3-D volume visualization of cysts is done by using the data in the cyst volume report of all input CT slice images. Figures 9 and 10 show the process of 3-D cyst volume visualization of a single

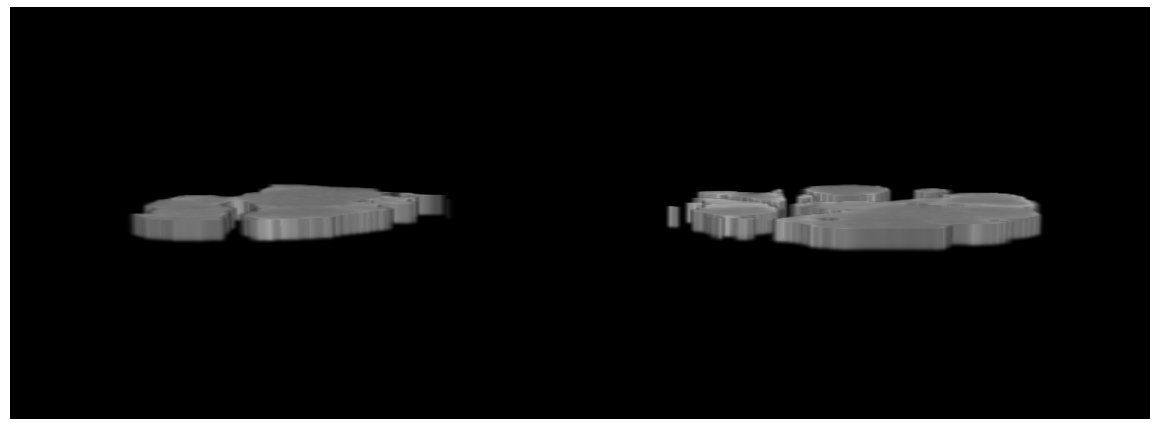

(a)

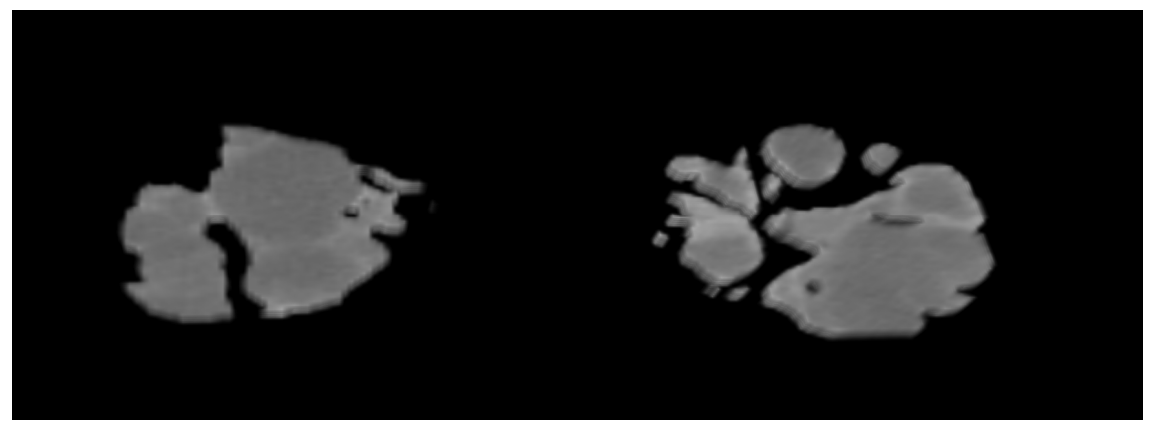

(b)

Figure 9. Process of 3-D cyst volume visualization of a single CT slice image. (a) side view of 3-D cyst volume, (b) top view of 3-D cyst volume. The region in gray is the cyst reigon.

CT slice image and a set of CT slice images, respectively. This process uses the result of cyst volume calculation and cyst segmentation. Figure 9 shows the process of 3-D cyst volume visualization of a single CT slice image. Figure 9(a) shows the side view of 3-D cyst volume.

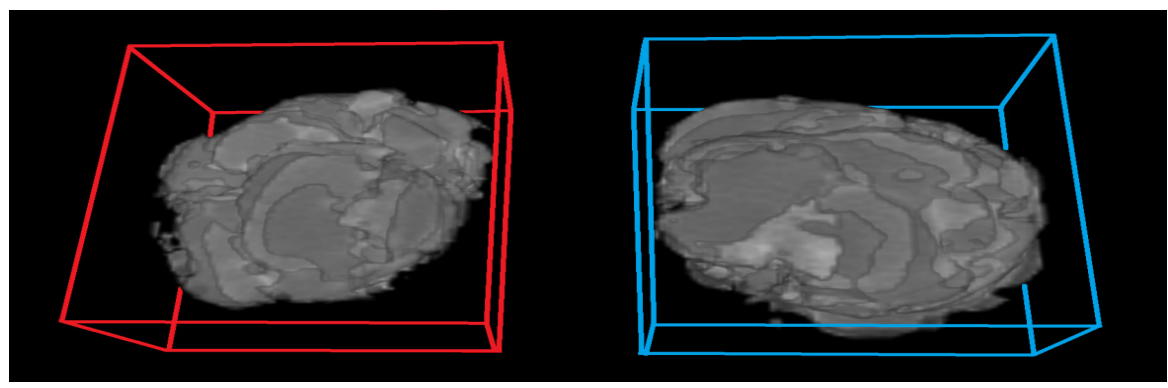

(a) 


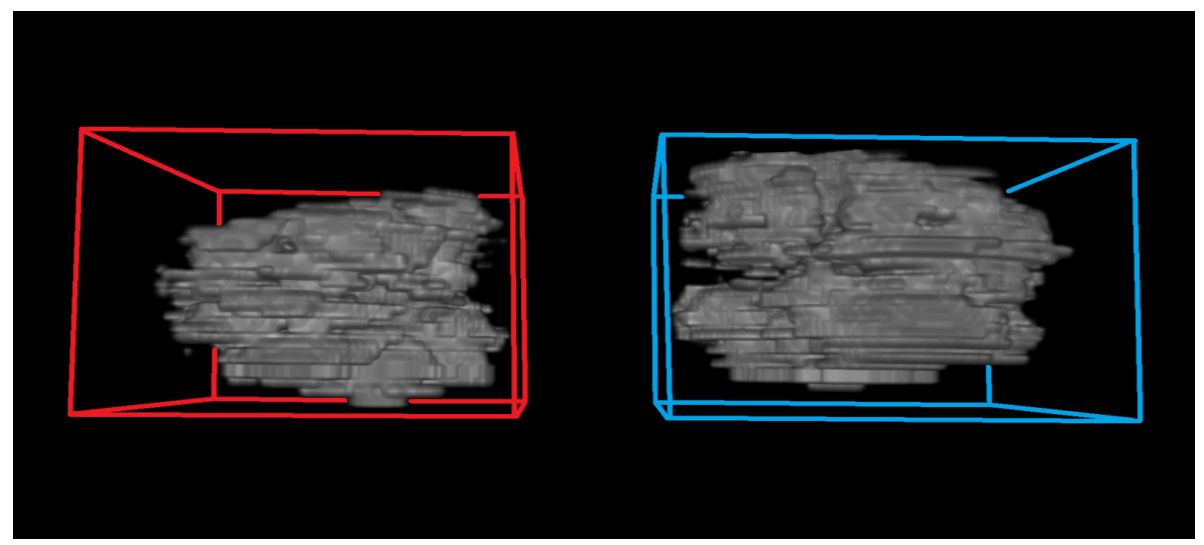

(b)

Figure 10. Process of 3-D cyst volume visualization. (a) top view of cyst volume, (b) side view of cyst volume. The hexahedron is the minimum bounding box that encloses the estimated cyst regions. The red and blue hexahedra contain cysts in the right kidney and left kidney, respectively.

Figure 9(b) shows the top view of 3-D cyst volume. The random distributions of cysts are observed more easily by 3 -D volume visualization than in the cyst volume report.

Figure 10 shows the 3 -D cyst volume visualization of a set of CT slice images. The inputs are a set of CT slice images of the same patient. The reconstructed 3-D model is based on the cyst volume report that includes the CT slice images with or without cysts. The distribution of cysts in each slice is different from each other, therefore, combining these scattered information from the cyst volume report can effectively explain the property of cysts. Figure 10(a) shows the top view of 3-D cyst volume, whereas Figure 10(b) shows the side view. Red and blue hexahedra are shown to illustrate minimum bounding boxes that enclose the estimated cysts in the right and left kidneys, respectively. As the results of 3-D volume visualization, the occupied volume and the distribution of cysts can be easily determined. This kind of visible information can help experts to estimate the state of illness and make reliable surgical plans.

\section{CONCLuSion}

In this paper, a segmentation method and a 3-D volume calculation method of cysts in kidney are proposed. First, cysts are separated from input CT slice images. 3-D volume visualization of cysts is performed by using the segmented cyst regions and calculated cyst volumes. Then, the distribution and the volume of cysts can be easily observed by 3-D volume visualization. Experimental results of the proposed method show a high accuracy of cyst volume calculation. Future work will focus on code optimization of the proposed method and resolution enhancement of 3-D volume visualization. 
International Journal of Computer Graphics \& Animation (IJCGA) Vol.5, No.1, January 2015

\section{ACKNOWLEDGMENTS}

This work was supported in part by the Brain Korea 21 Plus.

\section{REFERENCES}

[1] Y. M. Kwon, Y. H. Hwang, and Y. G. Jung, "Design and implementation of program for volumetric measurement of kidney," in Proc. Future Generation Information Technology, pp. 170-176, Gangneug, Korea, Dec. 2012.

[2] B. N. Li, C. K. Chui, S. Chang, and S. H. Ong, "Integrating spatial fuzzy clustering with level-set methods for automated medical image segmentation," Computers in Biology and Medicine, vol. 41, no. 1, pp. 1-10, Jan. 2011.

[3] B. N. Li, C. K Chui, S. Chang, and S. H. Ong, "A new unified level-set method for semi-automatic liver tumor segmentation on contrast-enhanced CT images," Expert Systems with Applications, vol. 39, no. 10, pp. 9661-9668, Aug. 2012.

[4] W. Burger and M. J. Burge, Digital Image Processing: An Algorithmic Introduction Using Java, New York, USA: Springer, 2008.

[5] N. Xu, N. Ahuja, and R. Bansal, "Object boundary segmentation using graph cuts based active contours," in Proc. IEEE Conf. Computer Vision and Pattern Recognition, vol. 2, pp. 46-53, Madison, WI, USA, Jun. 2003.

[6] R. Kaur, L. Kaur, and S. Gupta, "Enhanced k-mean clustering algorithm for liver image segmentation to extract cyst region," IJCA Special Issue on Novel Aspects of Digital Imaging Applications, pp. 5966, New York, USA, Jun. 2011.

[7] C.-C. Lee, S.-H. Chen, and Y.-C. Chiang, "Automatic liver diseases diagnosis for CT images using kernel-based classifiers,” in Proc. IEEE Conf. Automation Congress, pp. 1-5, Budapest, Hungary, Jul. 2006.

[8] H. Ling, S. K. Zhou, Y. Zheng, and B. Georgescu, "Hierarchical, learning-based automatic liver segmentation," in Proc. IEEE Conf. Computer Vision and Pattern Recognition, pp. 1-8, Anchorage, AL, USA, Jun. 2008.

[9] M. M. Sabry, C. B. Sites, A. A. Farag, S. Hushek, and T. Moriarty, "A fast automatic method for 3D volume segmentation of the human cerebrovascular," in Proc. CARS 2002 Computer Assisted Radiology and Surgery, pp. 382-387, Berlin, Germany, Jun. 2002.

[10] T. Heimann and H.-P. Meinzer, "Statistical shape models for 3D medical image segmentation: A review,” Medical Image Analysis, vol. 13, no. 4, pp. 543-563, Aug. 2009.

\section{Authors}

Nanzhou Piao received the B.S. degree in electronic engineering from Yanbian University of Science and Technology in 2011. Currently he is working toward the M.S. degree in electronic engineering from Sogang University. His current research interests are medical image analysis and face recognition.

Jong-Gun Kim received the B.S. degree in medical computer science from Eulji University in 2012 and the M.S. degree in electronic engineering from Sogang University in 2014. Currently he is working in Hyundai Mobis Co., Ltd., where he is involved in research and development of rear view camera system. His current interests are color processing and computer vision.

Rae-Hong Park received the B.S. and M.S. degrees in electronics engineering from Seoul National University, Seoul, Korea, in 1976 and 1979, respectively, and the M.S and Ph.D. degrees in electrical engineering from Stanford University, Stanford, CA, USA, in 1981 and 1984, respectively.

In 1984, he joined the faculty of the Department of Electronic Engineering, Sogang University, Seoul, Korea, where he is currently a Professor. In 1990, he spent his sabbatical year as a Visiting Associate Professor with the Computer Vision Laboratory, Center for Automation Research, University of Maryland 
at College Park, USA. In 2001 and 2004, he spent sabbatical semesters at Digital Media Research and Development Center (DTV image/video enhancement), Samsung Electronics Co., Ltd., Suwon, Korea. In 2012, he spent a sabbatical year in Digital Imaging Business (R\&D Team) and Visual Display Business (R\&D Office), Samsung Electronics Co., Ltd., Suwon, Korea. His current research interests are video communication, computer vision, and pattern recognition. He served as Editor for the Korea Institute of Telematics and Electronics (KITE) Journal of Electronics Engineering from 1995 to 1996.

Dr. Park was the recipient of a 1990 Post-Doctoral Fellowship presented by the Korea Science and Engineering Foundation (KOSEF), the 1987 Academic Award presented by the KITE, the 2000 Haedong Paper Award presented by the Institute of Electronics Engineers of Korea (IEEK), the 1997 First Sogang Academic Award, and the 1999 Professor Achievement Excellence Award presented by Sogang University. $\mathrm{He}$ is a co-recipient of the Best Student Paper Award of the IEEE Int. Symp. Multimedia (ISM 2006) and IEEE Int. Symp. Consumer Electronics (ISCE 2011). 